\title{
Free Androgen Index
}

National Cancer Institute

\section{Source}

National Cancer Institute. Free Androgen Index. NCI Thesaurus. Code C124341.

A measurement of androgen status in a biological specimen, which is calculated by dividing total testosterone by sex hormone binding globulin and multiplying by a constant (usually 100). 